\title{
Advances in Contactless Silicon Defect and Impurity Diagnostics Based on Lifetime Spectroscopy and Infrared Imaging
}

\author{
Jan Schmidt, Peter Pohl, Karsten Bothe, and Rolf Brendel \\ Institut für Solarenergieforschung Hameln/Emmerthal (ISFH), Am Ohrberg 1, 31860 Emmerthal, Germany
}

Received 19 March 2007; Accepted 12 April 2007

Recommended by Armin G. Aberle

\begin{abstract}
This paper gives a review of some recent developments in the field of contactless silicon wafer characterization techniques based on lifetime spectroscopy and infrared imaging. In the first part of the contribution, we outline the status of different lifetime spectroscopy approaches suitable for the identification of impurities in silicon and discuss-in more detail-the technique of temperature- and injection-dependent lifetime spectroscopy. The second part of the paper focuses on the application of infrared cameras to analyze spatial inhomogeneities in silicon wafers. By measuring the infrared signal absorbed or emitted from lightgenerated free excess carriers, high-resolution recombination lifetime mappings can be generated within seconds to minutes. In addition, mappings of non-recombination-active trapping centers can be deduced from injection-dependent infrared lifetime images. The trap density has been demonstrated to be an important additional parameter in the characterization and assessment of solar-grade multicrystalline silicon wafers, as areas of increased trap density tend to deteriorate during solar cell processing.
\end{abstract}

Copyright (C) 2007 Jan Schmidt et al. This is an open access article distributed under the Creative Commons Attribution License, which permits unrestricted use, distribution, and reproduction in any medium, provided the original work is properly cited.

\section{INTRODUCTION}

The performance of today's commercially produced silicon solar cells is to a great extent limited by recombination via defects and impurities in the bulk. This holds in particular for solar cells produced on multicrystalline silicon (mc-Si) wafers, containing large concentrations of crystallographic defects, such as dislocations and grain boundaries, as well as recombination-active impurities, such as transition metals. Despite the fact that the concentrations of these impurities are usually extremely low (typically well below $10^{13} \mathrm{~cm}^{-3}$ ), their impact on cell performance can be quite detrimental already above ultra low concentrations of $\sim 10^{11} \mathrm{~cm}^{-3}$. Such low impurity concentrations are not detectable by most analytical techniques. Only a few electrical techniques, such as capacitance-based deep-level transient spectroscopy (DLTS) [1] and lifetime spectroscopy (LS) [2-6], are capable of detecting and identifying electrically active impurities at concentrations below $10^{13} \mathrm{~cm}^{-3}$. The main advantages of LS over DLTS are that (i) it is a contactless technique, facilitating the sample preparation, and (ii) it is most sensitive to those centers that contribute to the total recombination rate (i.e., those centers that are actually relevant to solar cells). In contrast, standard DLTS only detects capture and emission of one type of carrier and gives, hence, in general no information on the recombination activity of the detected impurity. In the first part of this paper, we give a brief review of the different approaches to extract the characteristic defect parameters (i.e., energy level and electron-to-hole capture cross-section ratio) from LS measurements and address difficulties and limitations of the techniques.

Typically, the spatial distribution of recombination centers in mc-Si wafers is very inhomogeneous, requiring the application of spatially resolved measurement techniques. Traditionally, this is done by tedious point-by-point measurement techniques, such as the microwave-detected photoconductance decay (MW-PCD) $[7,8]$ technique, requiring typical measurement periods in the range of hours to obtain a high-resolution lifetime mapping. Recently, infraredcamera-based measurement techniques have been introduced $[9,10]$ which allow to generate high-resolution lifetime mappings within seconds to minutes. The principal idea of infrared lifetime mapping/carrier density imaging (ILM/CDI) is to make use of the free-carrier absorption or emission of sub-bandgap infrared photons. Using a suitable calibration, the spatial distribution of the light-generated 
excess carrier concentration is directly obtained from the infrared absorption or emission image recorded by the camera. Provided that the photogeneration is homogeneous over the entire wafer area, a recombination lifetime mapping can be deduced directly from the excess carrier concentration image. In addition to the spatial analysis of recombination centers, the infrared camera also allows to analyze the spatial distribution of non-recombination-active minority-carrier trapping centers. This infrared trap mapping (ITM) method [11] is of particular relevance to mc-Si wafers, as the trapdensity mapping predicts how a multicrystalline silicon wafer behaves during high-temperature processing. The second part of this review presents the current state of these infraredcamera-based measurement techniques, which-due to their short data acquisition times-evolve into in-line characterization tools for solar cell production lines in the near future.

\section{LIFETIME SPECTROSCOPY}

Figure 1 shows a comparison of the basic physical principles of LS and DLTS. While lifetime spectroscopy is based on measurements of recombination events via defect/impurity levels in the bandgap capturing electrons as well as holes, in standard DLTS measurements the detected defect level interacts only with the majority carriers (in Figure 1 with electrons), making it only sensitive to defect levels in the majority-carrier half of the bandgap. A major problem of DLTS measurements is that, despite the fact that energy level, majority-carrier capture cross-section, and defect concentration can be extracted from those measurements, no information on the recombination activity of the detected center is gained. This important device-relevant information is inherently obtained from lifetime spectroscopy measurements, which are particularly sensitive to such types of defects that actually result in recombination. Different variants of lifetime spectroscopy have been studied in the past of which we will give a brief overview in the following.

\subsection{Temperature-dependent lifetime spectroscopy}

Temperature-dependent lifetime spectroscopy (TDLS) measures the carrier lifetime of a sample under low-level injection conditions ( $\left.\tau^{\mathrm{LLI}}\right)$ and determines the defect energy level $E_{t}$ from the linear slope of the Arrhenius plot of $\ln \left(\tau^{\mathrm{LLI}} / T\right)$ versus $1 / T[2,4]$. However, a basic assumption of the standard TDLS analysis is that the electron and hole capture cross-sections $\sigma_{n}$ and $\sigma_{p}$ are independent of temperature, which is not valid in general. Moreover, the standard TDLS analysis does not allow to decide whether the defect energy level is located in the upper or in the lower half of the bandgap. The TDLS analysis has been improved by enlarging the temperature range down to temperatures well below room temperature and by fitting the complete temperaturedependent lifetime curve [6] using the Shockley-Read-Hall (SRH) equation $[12,13]$. This allows an improved determination of the energy level.

\subsection{Injection-dependent lifetime spectroscopy}

Less frequently applied in the past has been the technique of injection-dependent lifetime spectroscopy (IDLS) since in most cases it results only in a relatively broad uncertainty range for $E_{t}$ [3]. On the other hand, IDLS is capable of determining the $\sigma_{n} / \sigma_{p}$ ratio with a better accuracy than TDLS. IDLS measurements on silicon wafers with varying doping concentrations is one possibility to narrow down the uncertainty range for $E_{t}[3]$. However, this approach requires many different samples with lifetimes limited by the same type of defect.

\subsection{Temperature- and injection-dependent lifetime spectroscopy}

In order to combine the advantages of TDLS and IDLS, we have recently introduced temperature- and injectiondependent lifetime spectroscopy (TIDLS) [5]. Our experimental set-up is based on an extended quasi-steady-state photoconductance (QSSPC) system [5, 14]. Importantly, TIDLS not only determines the defect energy level with improved accuracy, but also reveals the temperature dependence of the capture cross-sections and permits the distinction between shallow and deep-level centers. Despite its large potential for the identification of efficiency-limiting defects in solar-grade silicon materials, TIDLS is still not widely used by the PV community. This is mainly due to the fact that the data evaluation is relatively complex because it requires the simultaneous fitting of the SRH equation to a large number of injection-dependent lifetime curves measured at varying temperatures. The results of such fitting attempts are usually ambiguous, imposing an additional complication to the general analysis of TIDLS measurements. In an effort to facilitate the application of TIDLS, we have recently simplified the analysis of temperature- and injection-dependent lifetime measurements [15].

\subsection{Simplified TIDLS analysis}

Our simplified analysis determines the energy level $E_{t}$ and the ratio of the electron-to-hole capture cross-sections $k=\sigma_{n} / \sigma_{p}$ of the lifetime-limiting recombination center without the requirement of a simultaneous fitting of all measured $\tau(\Delta n, T)$ curves. The simplified analysis is restricted to recombination centers which - at a characteristic temperature $T_{c}$ - change their $\tau(\Delta n)$ slope with increasing temperature from positive to negative. As the experimentally accessible temperature range of our TIDLS set-up is restricted to the temperature range $30-170^{\circ} \mathrm{C}$, we are only capable of analyzing defect levels with characteristic temperatures $T_{c}$ within this range. This restricts our analysis to recombination centers with a distance from one of the band edges within the range of $0.2-$ $0.4 \mathrm{eV}$, as estimated using the SRH equation [15].

In order to demonstrate our simplified TIDLS analysis, we have applied it to an intentionally tungsten-contaminated p-type Czochralski-grown silicon (Cz-Si) wafer of doping concentration $N_{\text {dop }}=4.7 \times 10^{14} \mathrm{~cm}^{-3}$. Both wafer surfaces are passivated with plasma-enhanced chemical vapor 


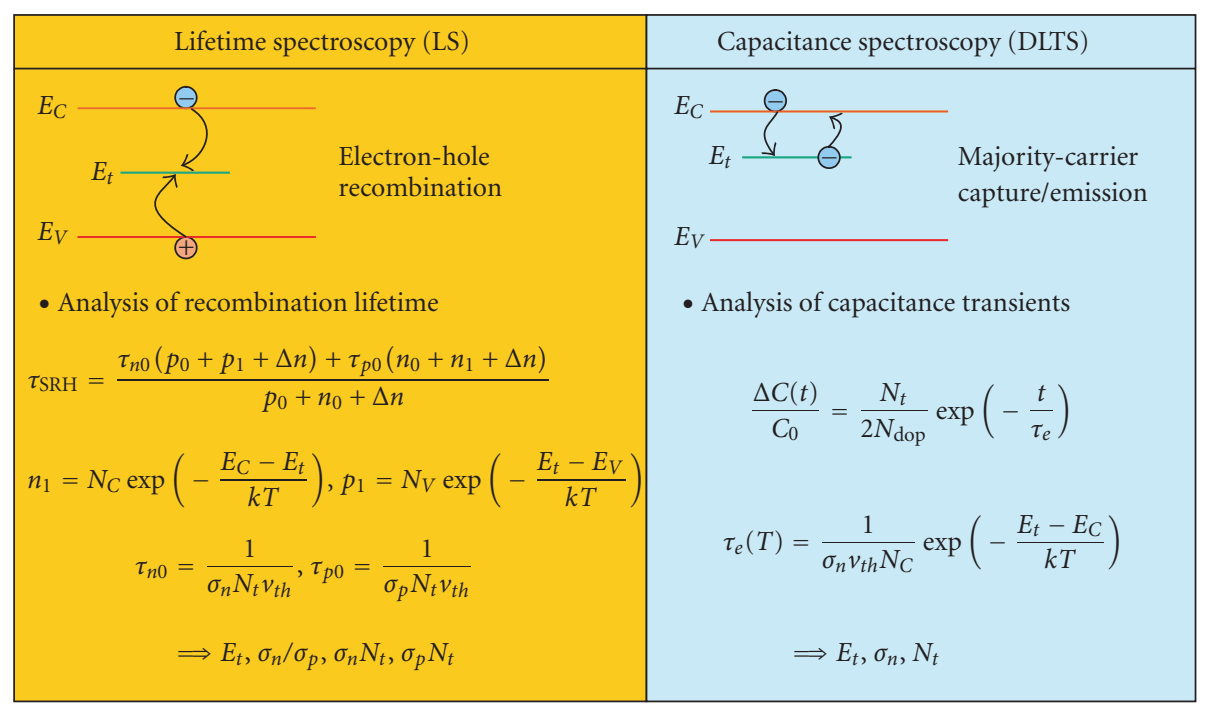

FIGURE 1: Comparison of the physical principles of lifetime spectroscopy (LS) and capacitance-based DLTS.

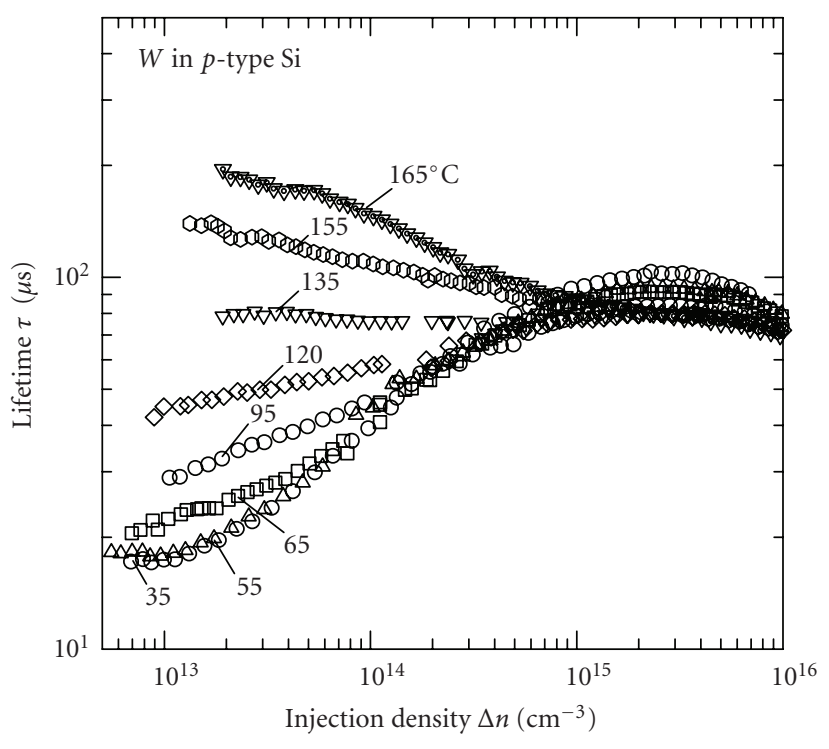

FIgURE 2: Temperature- and injection-dependent carrier lifetime measurements of a tungsten-contaminated $p$-type $\mathrm{Cz}$-Si wafer of doping concentration $N_{\text {dop }}=4.7 \times 10^{14} \mathrm{~cm}^{-3}$.

deposited silicon nitride films resulting in surface recombination velocities well below $10 \mathrm{~cm} / \mathrm{s}$ [16]. Due to the excellent passivation properties of our silicon nitride films, we neglect any surface recombination and identify the measured effective lifetimes with the bulk lifetimes of the wafer under test. TIDLS measurements have been performed in the temperature range between 30 and $165^{\circ} \mathrm{C}$ in steps of $5^{\circ} \mathrm{C}$ using a QSSPC set-up extended with a temperature-controlled sample stage [5, 14]. Figure 2 shows a selection of $\tau(\Delta n)$ curves measured at different temperatures. By merely looking at the qualitative $\tau(\Delta n, T)$ behavior, several important features of the lifetime-limiting recombination center-in this case interstitial tungsten $\left(W_{i}\right)$ —can be deduced.

First, for temperatures up to $60^{\circ} \mathrm{C}$ the injection-dependent lifetime curves do not change at all. This implies that for low temperatures the recombination center behaves like a deep-level center. As a consequence, we can immediately calculate the capture cross-section ratio $k$ using the equation [15]

$$
k=\left(\frac{\tau_{\eta}}{\tau_{n 0}}-1\right)\left(\eta^{-1}+1\right),
$$

which we have derived from the SRH equation. In (1), $\eta \equiv$ $\Delta n / N_{\text {dop }}$ is the injection level, $\tau_{n 0}$ is the low-injection lifetime, and $\tau_{\eta}$ is the lifetime measured at an intermediate injection level $\eta$, the latter being typically chosen between 0.1 and 1.0. As can be seen from (1), the determination of $k$ requires only two lifetime values, one measured under low-injection conditions, the second one measured at an intermediate injection level.

However, to determine the capture cross-section ratio $k$ with improved accuracy, we have determined $k$ at different injection levels $\eta$ and for varying temperatures. Figure 3 shows the results for the $k(\eta)$ curve calculated from the measured $\tau(\Delta n)$ dependence at $35^{\circ} \mathrm{C}$. As expected for a singlelevel defect, the analysis results in a constant $k$, in the present case $k \approx 8$. This procedure can also be regarded as a test for the applicability of the single-level defect recombination theory, which is only valid if no $k(\eta)$ dependence is observed. The $k$ ratio decreases slightly for injection levels above unity (not shown in Figure 3). This can be attributed to an additional shallow defect level leading to a decreasing lifetime for higher injection levels. Applying (1) to all injection-dependent lifetime curves measured in the temperature range between 30 and $60^{\circ} \mathrm{C}$ results in a more accurate capture cross-section ratio and a corresponding uncertainty range of $k=8.5 \pm 1.0$. In the following analysis, we assume 
this $k$ value to be temperature-independent, that is, electron and hole capture cross-sections are assumed to have the same temperature dependence.

The second important feature of the measured $\tau(\Delta n, T)$ curves shown in Figure 2 is that at injection densities below the doping concentration, the slope of the injectiondependent lifetime curves changes from positive to negative at $135^{\circ} \mathrm{C}$. This characteristic temperature of $T_{c}=408 \mathrm{~K}$ can be determined with an accuracy of $\pm 5 \mathrm{~K}$ without any fitting. The energy level $E_{t}$ of the recombination center can now directly be calculated from the measured $T_{c}$.

However, the solution is not unambiguous because we have to consider two different cases: (i) $E_{t}$ is located in the lower half of the silicon bandgap and (ii) $E_{t}$ is located in the upper bandgap half. To derive the relation between $E_{t}$ and $T_{c}$, we consider at first case (i). Using the analytical expression given by the SRH equation for the $\tau(\Delta n, T)$ dependence and assuming that at $T_{c}$ the slope $\partial \tau / \partial \Delta n$ vanishes, we obtain the expression [15]

$$
E_{t}-E_{V}=-k_{B} T_{c} \ln \left[k \frac{N_{\mathrm{dop}}}{N_{V}^{300 \mathrm{k}}}\left(\frac{300 \mathrm{k}}{T_{c}}\right)^{3 / 2}\right],
$$

where $k_{B}$ is Boltzmann's constant and $N_{V}^{300 \mathrm{k}}$ is the effective density of states in the valence band at $300 \mathrm{~K}$. Using the experimentally determined values of $T_{c}=(408 \pm 5) \mathrm{K}$ and $k=8.5 \pm 1.0$, we determine an energy level of $E_{t}-E_{V}=$ $(0.33 \pm 0.01) \mathrm{eV}$.

Assuming case (ii) we obtain a slightly different equation for $E_{t}[15]$ :

$$
E_{C}-E_{t}=-k_{B} T_{c} \ln \left[\frac{N_{\mathrm{dop}}}{N_{C}^{300 \mathrm{k}}}\left(\frac{300 \mathrm{~K}}{T_{c}}\right)^{3 / 2}\right]
$$

with $N_{C}^{300 \mathrm{k}}$ being the effective density of states in the conduction band at $300 \mathrm{~K}$. Interestingly, (2) and (3) differ slightly as the energy level calculated from (2) depends on $k$, whereas the energy level calculated from (3) does not depend on the capture cross-section ratio. As a consequence, the energy levels may differ significantly if the capture cross-sections are strongly asymmetric, which is frequently the case. Using (3) with the measured characteristic temperature of $T_{c}=(408 \pm$ 5) $\mathrm{K}$, we obtain an energy level of $E_{C}-E_{t}=(0.40 \pm 0.01) \mathrm{eV}$.

Due to the strong asymmetry in the capture cross-section ratio, this energy level differs considerably from the energy level determined using (2). In the next step of the analysis, it has to be decided which of the two calculated energy levels is the true one. This can simply be done by performing a standard TDLS analysis at a well-defined injection density. Figure 4 shows an Arrhenius plot of the carrier lifetimes measured at $\Delta n=5 \times 10^{13} \mathrm{~cm}^{-3}$. The lifetime data measured above $\sim 100^{\circ} \mathrm{C}$ shows a clear linear dependence in the Arrhenius plot of Figure 4, resulting in an energy level of $\Delta E_{t}=(0.31 \pm 0.02) \mathrm{eV}$. From the standard TDLS analysis alone it cannot be decided if this energy level is located in the upper or in the lower bandgap half. However, by comparing the result of the standard TDLS analysis with our above analysis of the temperature- and injection-dependent

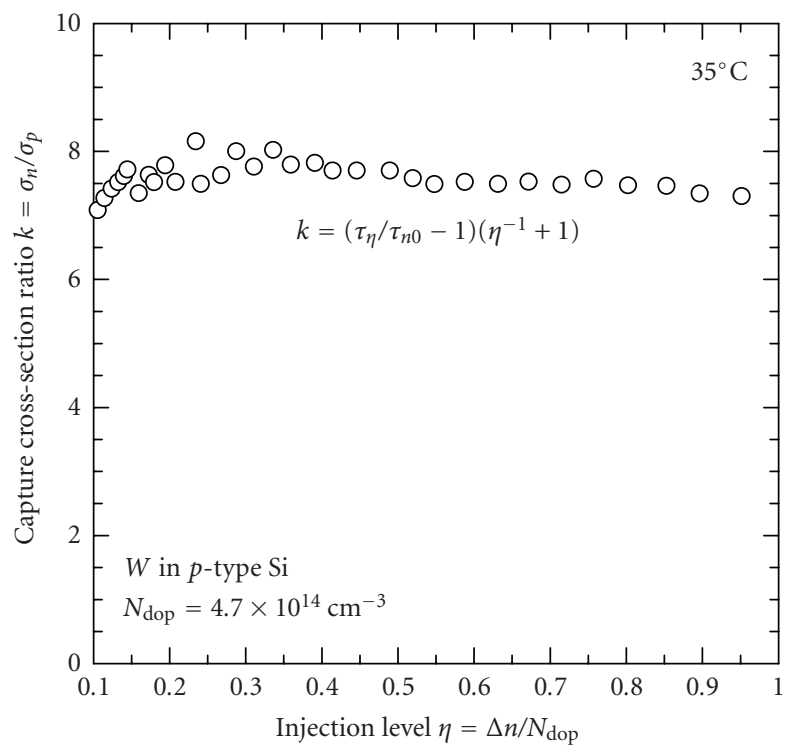

FIGURE 3: Capture cross-section ratio $k=\sigma_{n} / \sigma_{p}$ as a function of injection level $\eta$ at $35^{\circ} \mathrm{C}$, as calculated from the corresponding $\tau(\Delta n)$ curve in Figure 2.

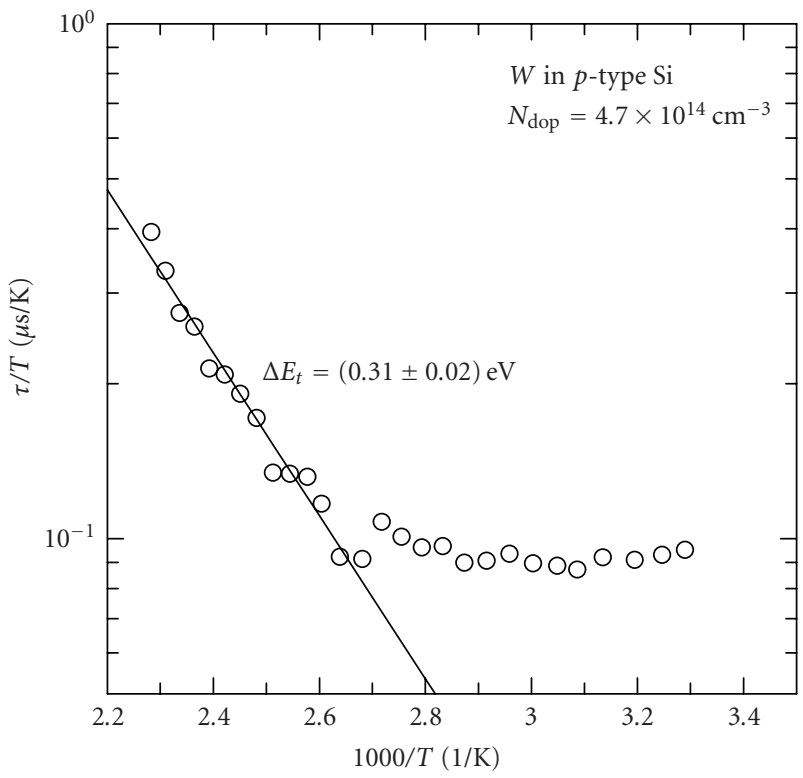

Figure 4: Arrhenius plot of the lifetime divided by temperature $\tau / T$. The lifetime data has been extracted from Figure 2 at an injection density of $\Delta n=5 \times 10^{13} \mathrm{~cm}^{-3}$. From the linear part of the curve, an energy level of $\Delta E_{t}=0.31 \mathrm{eV}$ is determined.

lifetime measurements, it can be concluded that the true energy level is located in the lower half of the silicon bandgap at $E_{t}-E_{V}=(0.33 \pm 0.01) \mathrm{eV}$. This result is in excellent agreement with literature data for the energy level of interstitial tungsten in silicon $[17,18]$. It is intriguing that energy level and capture cross-section ratio can both be determined with 
a very high degree of accuracy without the necessity of any complex fitting routines using the simplified TIDLS evaluation method. For comparison we have also fitted all TIDLS curves simultaneously using the SRH equation. This procedure results in an energy level of $E_{t}-E_{V}=(0.32 \pm 0.02) \mathrm{eV}$ and a $k$ value of $\sim 10$, which is also in excellent agreement with the result of our simplified TIDLS analysis.

\section{INFRARED-CAMERA-BASED CHARACTERIZATION TECHNIQUES}

Since the most common silicon wafer material used today in solar cell production is multicrystalline silicon with strongly inhomogeneous lateral distributions of defects and impurities, characterization techniques giving spatial information are indispensable. In the past, several contactless lifetime mapping techniques have been applied to characterize inhomogeneities in silicon wafers. Two prominent examples of such techniques are MW-PCD $[7,8]$ and modulated free-carrier absorption (MFCA) [19]. Both techniques generate the maps from point-by-point measurements, requiring typical measurement periods of hours for recording high-resolution $(\sim 200 \mu \mathrm{m})$ lifetime maps. To decrease the long measurement periods, infrared (IR)-camera lifetime mapping/carrier density imaging (ILM/CDI) has been introduced recently $[9,10]$. Using an IR camera (i.e., an array of IR detectors) sensitive in the wavelength range between $\sim 3$ and $8 \mu \mathrm{m}$ instead of a single detector reduces the measurement period for a high-resolution mapping from hours to minutes and sometimes even seconds. Note that IR cameras can also be used to image temperature distributions on solar cells, which has been successfully applied as "lock-in infrared thermography" to generate mappings of shunts or series resistances of solar cells $[20,21]$. More recently, camera-based photoluminescence imaging has been introduced, which also allows a very fast imaging of silicon wafers and solar cells at high resolution using a conventional relatively cheap silicon CCD camera [22]. A detailed discussion of all camera-based characterization techniques currently under investigation is however beyond the scope of this review paper.

\subsection{Infrared lifetime mapping}

Figure 5 shows a schematic of the ILM/CDI measurement principle. The technique is based on measuring the freecarrier absorption $[9,10]$ or, equivalently, the free-carrier emission $[9,23]$ of sub-bandgap photons. In order to improve the sensitivity, lock-in technique is applied. For further technical details the interested reader is referred to [24]. In this review, we focus on the basic measurement principle only. One image is recorded with above-bandgap illumination of the sample and a second one without illuminating the wafer. The difference image is proportional to the light-generated excess carrier concentration. The calibration is performed by recording the IR emission signal of several silicon wafers of known doping concentrations. The proportionality factor $m$ between the camera signal and the freecarrier concentration times the wafer thickness is obtained

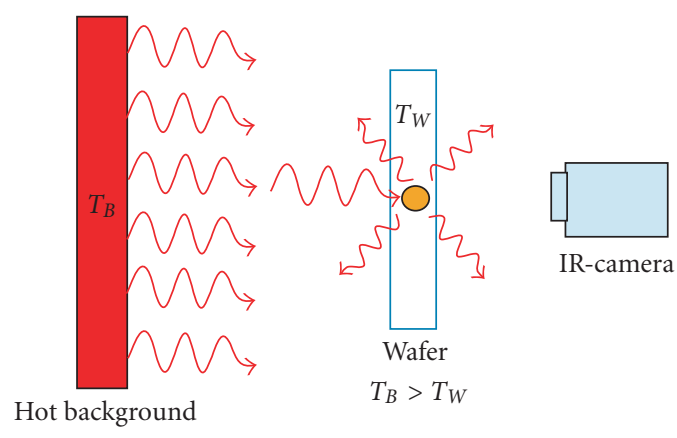

(a) Absorption ILM

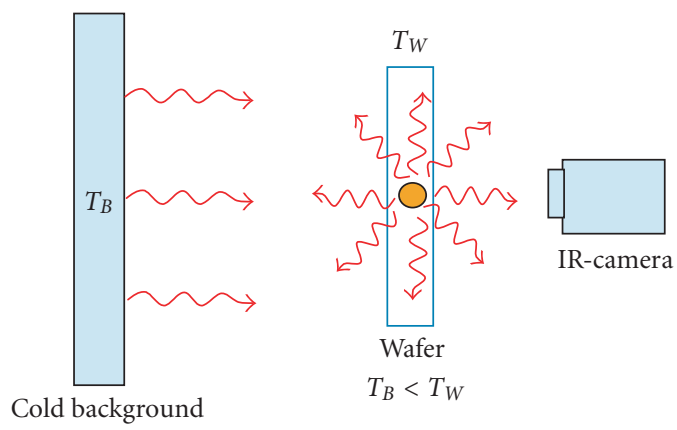

(b) Emission ILM

FIGURE 5: Schematic of the infrared lifetime mapping/carrier density imaging (ILM/CDI) principle: (a) absorption mode, (b) emission mode.

from a linear fit to the measured data. Using the measured photogeneration rate $G$ of the excitation light, the lifetime mapping $\tau(x, y)$ is calculated directly from the IR emission signal image $S(x, y)$ using the equation [9]

$$
\tau(x, y)=\frac{S(x, y)}{m\left(1+\alpha_{n} / \alpha_{p}\right) G W}
$$

with $W$ being the wafer thickness and $\alpha_{n / p}$ being the absorption coefficient of free electrons/holes at the detection wavelength. The correction factor $\left(1+\alpha_{n} / \alpha_{p}\right)$ accounts for the fact that under illumination, electrons as well as holes are generated, whereas in our calibration procedure only $p$-type silicon material is used $[9,25]$. According to Kirchhoff's law, the absorption always equals the emission. Hence, the measurement can be performed in absorption or emission mode, depending on the temperature of the material under investigation and the temperature of the background, as shown in Figure 5. The exemplary measurements on mc-Si wafers presented in Section 3.3 are carried out in emission mode.

\subsection{Infrared trap mapping}

In multicrystalline silicon wafers as well as in Czochralskigrown monocrystalline silicon wafers, abnormally high lifetimes are frequently measured at low injection levels. This effect has been assigned to the presence of non-recombinationactive minority-carrier trapping centers [26-28]. At excess 
carrier concentrations comparable to or below the trap density, charge neutrality implies that the presence of minoritycarrier traps causes a relative increase in the concentration of majority carriers, resulting in an increase of the steadystate photoconductance signal measured using QSSPC and the free-carrier absorption/emission signal recorded in ILM measurements. In a recent contribution, [11] we have shown that trap concentration and energy level mappings can be generated from ILM mappings recorded at different illumination intensities. This infrared trap mapping (ITM) technique is based on fitting the adapted Hornbeck-Haynes equation $[11,29]$ for the "apparent" lifetime

$$
\tau_{a}=\left(1+\frac{N_{t}}{\left(\Delta n+N_{t}\left(\tau_{t} / \tau_{d}\right)\right)} \frac{\alpha_{p}}{\left(\alpha_{p}+\alpha_{n}\right)}\right) \tau_{r}
$$

to the measured lifetime data at each point of the infrared lifetime mapping. In (5), $\tau_{r}$ is the recombination lifetime, $N_{t}$ the trap density, and $\tau_{t} / \tau_{d}$ the ratio of trapping-todetrapping time constants. The latter quantity is directly related to the energy level of the trap. For $n$-type material, $\alpha_{p}$ in the numerator has to be replaced by $\alpha_{n}$. As our measurements are performed under low-injection conditions, $\tau_{r}$ does not depend on the injection level. Moreover, the apparent excess carrier concentration $\Delta n_{a}$ is calculated via the expression $\Delta n_{a}=\left(\tau_{a} / \tau_{r}\right) \Delta n$ [28]. A suppression of the trapping effect can be realized by illuminating the wafer with sub-bandgap illumination in addition to the excess-carriergenerating light during the ILM measurement [30], which enables the extraction of the recombination lifetime from ILM measurements even at very low injection levels.

\subsection{Exemplary measurements on mc-Si wafers}

\subsubsection{As-grown state}

Figure 6 shows two lifetime images $\tau(x, y)$ of an mc-Si sample recorded at two different illumination intensities $\left(3 \times 10^{-3}\right.$ suns and 1 sun) [31]. Due to minority-carrier trapping the area-averaged apparent lifetime of $\tau_{a}=350 \mu$ s determined at $3 \times 10^{-3}$ suns [Figure $6(\mathrm{a})$ ] is much larger than the areaaveraged lifetime of 110 microseconds at 1 sun [Figure 6(b)], where trapping effects are negligible and thus the actual recombination lifetime is measured. As can be seen from Figure 6, the two lifetime mappings do not correlate perfectly. Apparently, additional information on the spatial distribution of the trapping centers is provided by the lifetime image measured at an illumination intensity of $3 \times 10^{-3}$ suns.

Fitting (5) to the lifetime images recorded at different illumination intensities results in the $N_{t}$ image shown in Figures 7(a). Figure 7(b) shows a photograph of the wafer after applying a Dash etch [32] to reveal a possible correlation of the traps with dislocations. Indeed, the spatial distribution of dislocations shown in Figure 7(b) is in excellent agreement with the trap density distribution in Figure 7(a).

\subsubsection{Impact of phosphorus gettering}

Figure 8 shows a comparison of the recombination lifetime maps recorded at an injection density of $\Delta n=10^{14} \mathrm{~cm}^{-3}$ be-

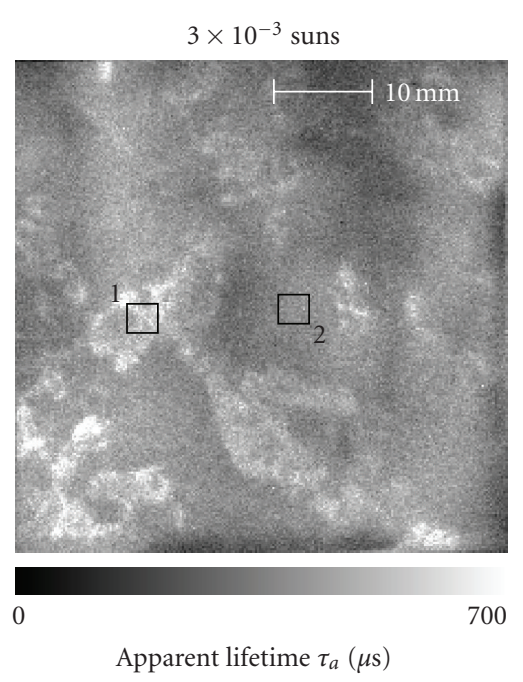

(a)

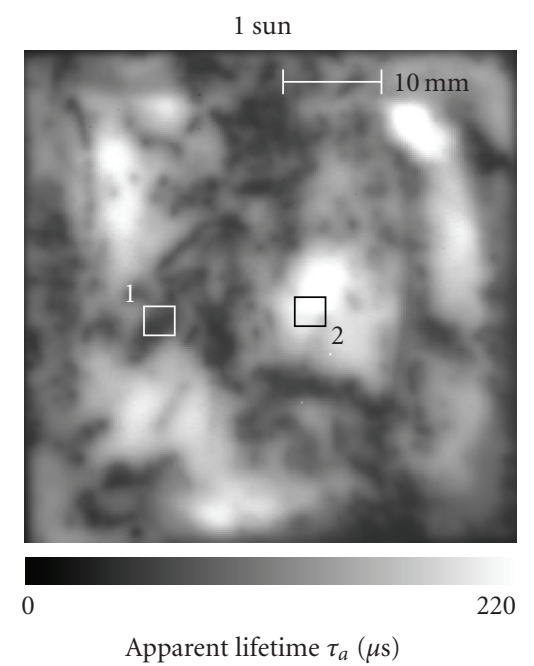

(b)

FIGURE 6: Infrared lifetime mappings of an mc-Si wafer measured at two different illumination intensities: (a) $3 \times 10^{-3}$ suns and (b) 1 sun.

fore and after phosphorus gettering [31]. The area-averaged recombination lifetime increases from 51.9 microseconds before gettering to 65.3 microseconds after gettering. However, $\tau_{r}$ increases in particular in those regions which already had the highest lifetimes before gettering. In regions of low lifetimes before gettering, the recombination lifetime shows only a small improvement or even decreases.

Figure 9 shows injection-dependent lifetime curves $\tau_{a}\left(\Delta n_{a}\right)$ measured at two different positions on the wafer indicated in Figures 6-8. The two positions correspond to areas of highest and lowest trap densities. At position 1, we determine $N_{t}=2.1 \times 10^{13} \mathrm{~cm}^{-3}$ and at position $2, N_{t}=$ $9.0 \times 10^{12} \mathrm{~cm}^{-3}$. The injection-dependent lifetime curves in Figure 9 show that for injection densities above $1 \times 10^{14} \mathrm{~cm}^{-3}$ 


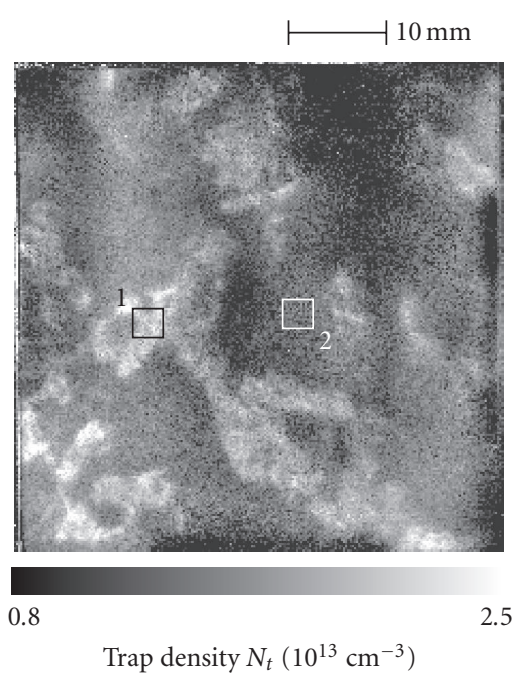

(a)

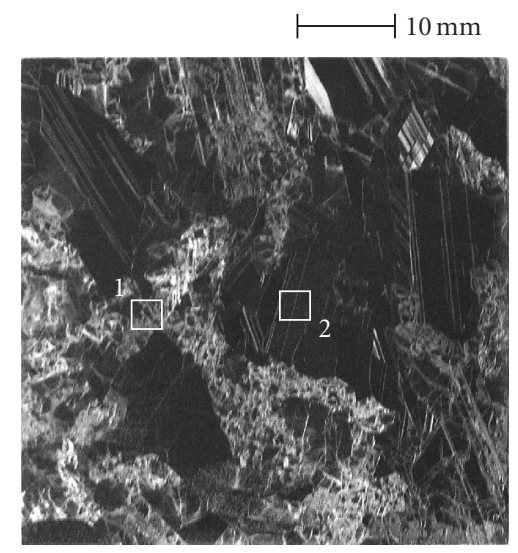

(b)

Figure 7: (a) Trap density image $N_{t}(x, y)$ of the mc-Si wafer shown in Figure 6. (b) Photograph of the same wafer after applying a Dash etch. Brighter regions correspond to regions of increased dislocation densities.

trapping effects are negligible and, thus, the actual recombination lifetime is obtained. The curve measured at position 2 clearly reveals an increase of the recombination lifetime after gettering, whereas at position $1, \tau_{r}$ decreases after the phosphorus gettering treatment.

Our interpretation of the observed behavior is that areas exhibiting higher dislocation densities (position 1) contain significant concentrations of metallic impurities (partly in the form of metal silicide precipitates), which are captured within the stress field surrounding a dislocation. These impurities are released during the high-temperature gettering treatment and contaminate the regions surrounding the dislocations [24]. This effect leads to a severe degradation in the solar cell performance in contrast to regions with lower dislocation densities (position 2), where the phosphorus gettering treatment mainly removes recombination-active interstitial

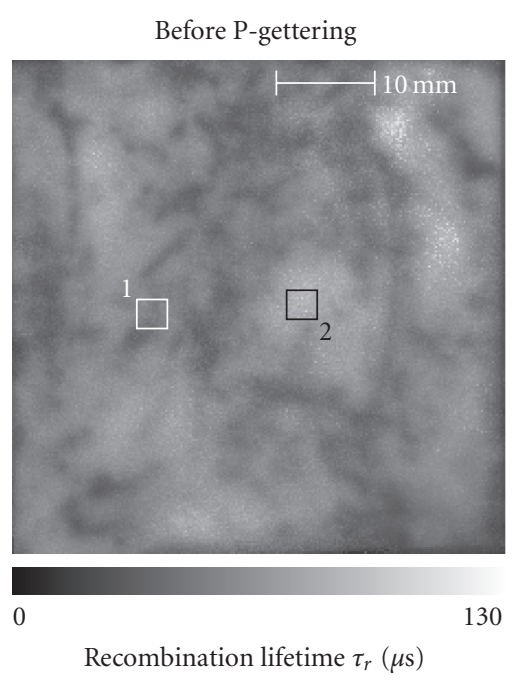

(a)

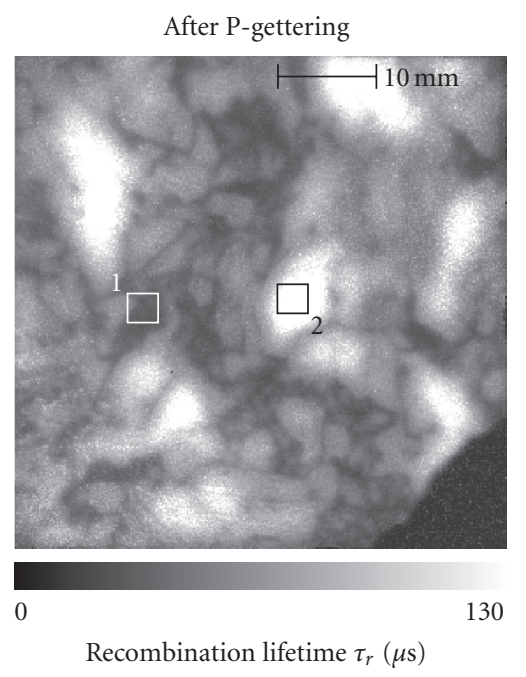

(b)

FIGURE 8: Recombination lifetime images $\tau_{r}(x, y)$ determined at an injection density of $\Delta n=10^{14} \mathrm{~cm}^{-3}$ as calculated using an automated fitting routine for the wafer shown in Figure 6 before (a) and after (b) phosphorus gettering.

metallic impurities. Note that more recently similar results have been published in a parallel study [33].

\subsubsection{Trap mapping without surface passivation}

As already mentioned in the previous section, the apparent lifetime image [Figure 6(a)] and the corresponding $N_{t}$ image [Figure $7(\mathrm{a})]$ show a good qualitative agreement, demonstrating that a single ILM measurement already reveals if trapping at dislocations is present and which regions of the wafer are affected. In order to facilitate a possible industrial application of IR-camera-based trap imaging, we have 


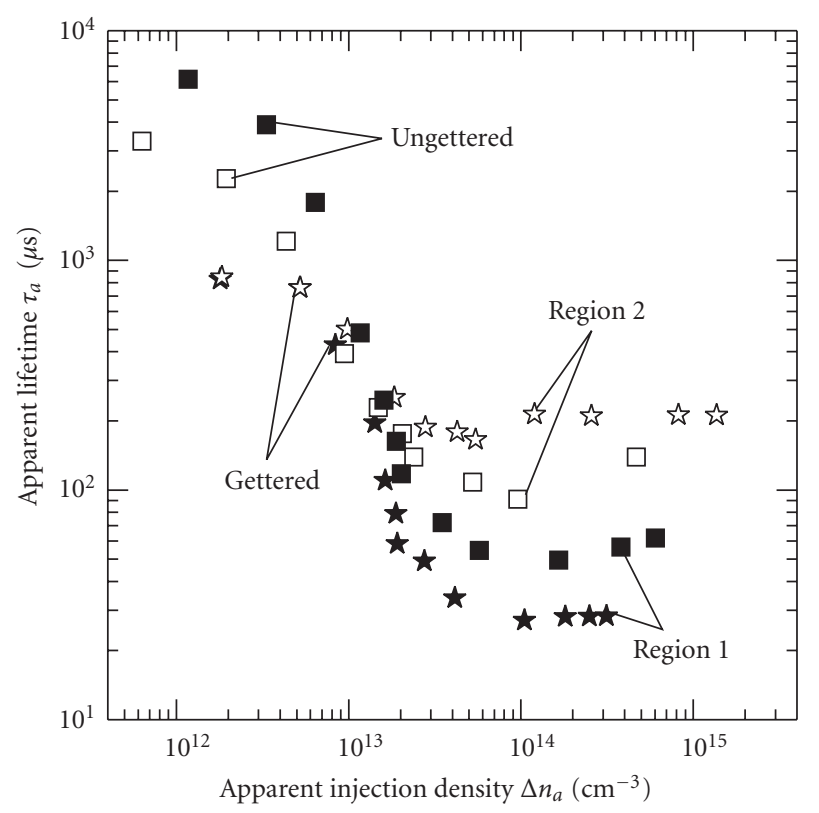

FIGURE 9: Injection-dependent lifetime curves $\tau_{a}\left(\Delta n_{a}\right)$ at two different positions (marked in Figures 6-8) of an mc-Si wafer. The wafer was measured before (squares) and after (stars) phosphorus gettering.

performed only one single IR emission measurement on ascut mc-Si wafers without applying any surface treatment [31]. Figure 10(a) shows the measured infrared emission picture $S(x, y)$ of an as-cut block-cast mc-Si wafer $(125 \times$ $125 \mathrm{~mm}^{2}$ ) recorded at $1 / 100$ suns. The measurement period was 20 minutes. The image reveals areas with increased dislocation densities located on the left-hand side, bottom side, and top right corner of the wafer. A comparison with the $N_{t}$ image obtained using the ITM method [see Figure 10(b)] shows that the two images agree well.

Our measurements suggest that regions of increased dislocation densities correlate with increased densities of metallic impurities, mostly in the form of precipitates [31]. A hightemperature step, such as the phosphorus gettering treatment, dissolves metal precipitates and degrades the lifetime in highly dislocated areas. Hence, the application of IR camera measurements to unpassivated as-cut mc-Si wafers helps to identify poorly getterable areas. However, a further reduction of the measurement time is required to enable the application of this technique to the front-end materials characterization in solar cell production lines.

\section{CONCLUSIONS}

Contactless characterization techniques enabling (i) a reliable identification of contaminants in silicon wafers and (ii) a fast determination of their spatial distribution are becoming increasingly important in silicon photovoltaics, since most commercially produced solar cells are still limited by defect-related recombination in the bulk. The potential of infrared-camera-based defect characterization techniques

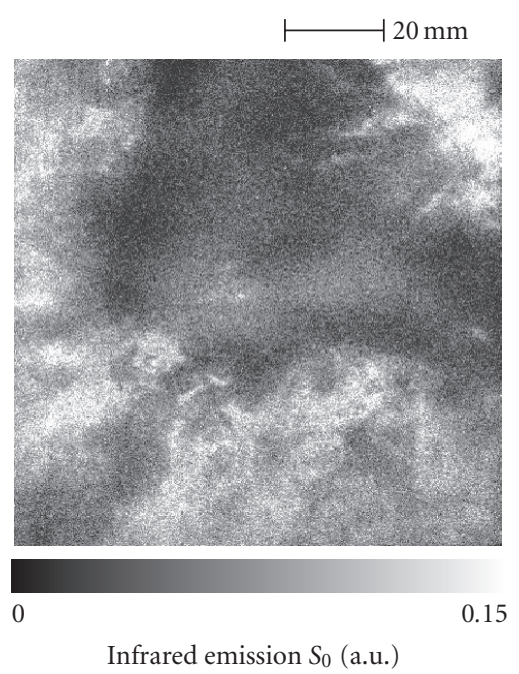

(a)

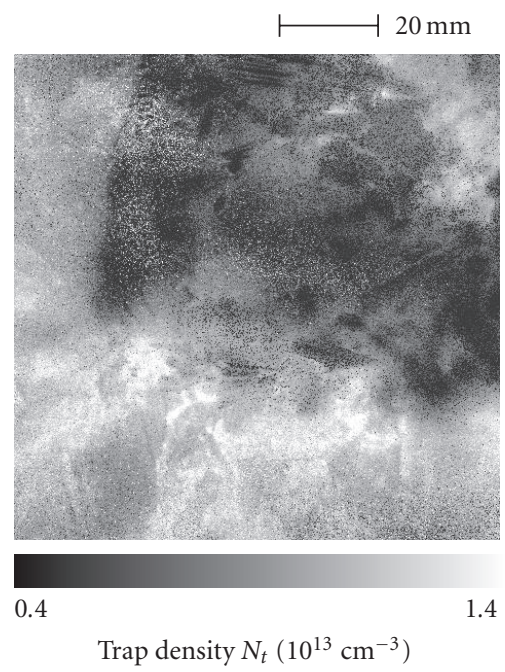

(b)

FIGURE 10: (a) Infrared emission signal $S(x, y)$ of a block-cast mc-Si wafer without any surface passivation measured at an illumination intensity of 0.01 sun. Brighter regions correspond to areas of increased trap densities. (b) Trap density image $N_{t}(x, y)$ of the same wafer after passivating both surfaces with silicon nitride.

has just started to be explored. Infrared trap mapping represents a new source of information on the defect structure of a silicon material. An important improvement to the analysis of trap parameters by quasi-steady-state photoconductance measurements is the possibility of examining spatial correlations between trapping and recombination centers as well as correlations with structural defects.

\section{ACKNOWLEDGMENTS}

The authors are grateful to T. Abe (ShinEtsu) for supplying tungsten-contaminated silicon wafers. Funding was provided by the German State of Lower Saxony. 


\section{REFERENCES}

[1] D. V. Lang, "Deep-level transient spectroscopy: a new method to characterize traps in semiconductors," Journal of Applied Physics, vol. 45, no. 7, pp. 3023-3032, 1974.

[2] Y. Hayamizu, T. Hamaguchi, S. Ushio, T. Abe, and F. Shimura, "Temperature dependence of minority-carrier lifetime in iron-diffused $\rho$-type silicon wafers," Journal of Applied Physics, vol. 69 , no. 5, pp. 3077-3081, 1991.

[3] J. Schmidt and A. Cuevas, "Electronic properties of lightinduced recombination centers in boron-doped Czochralski silicon," Journal of Applied Physics, vol. 86, no. 6, pp. 31753180, 1999.

[4] S. Rein, T. Rehrl, W. Warta, and S. W. Glunz, "Lifetime spectroscopy for defect characterization: systematic analysis of the possibilities and restrictions," Journal of Applied Physics, vol. 91, no. 3, pp. 2059-2070, 2002.

[5] J. Schmidt, "Temperature- and injection-dependent lifetime spectroscopy for the characterization of defect centers in semiconductors," Applied Physics Letters, vol. 82, no. 13, pp. 2178 2180, 2003.

[6] S. Rein, Lifetime Spectroscopy, Springer, Berlin, Germany, 2005.

[7] M. Kunst and G. Beck, "The study of charge carrier kinetics in semiconductors by microwave conductivity measurements," Journal of Applied Physics, vol. 60, no. 10, pp. 3558-3566, 1986.

[8] J. Schmidt and A. G. Aberle, "Accurate method for the determination of bulk minority-carrier lifetimes of mono- and multicrystalline silicon wafers," Journal of Applied Physics, vol. 81, no. 9, pp. 6186-6199, 1997.

[9] M. Bail, J. Kentsch, R. Brendel, and M. Schulz, "Lifetime mapping of Si wafers by an infrared camera [for solar cell production]," in Proceedings of the 28th IEEE Photovoltaic Specialists Conference, pp. 99-103, Anchorage, Alaska, USA, September 2000.

[10] S. Riepe, J. Isenberg, C. Ballif, S. W. Glunz, and W. Warta, "Carrier density and lifetime imaging of silicon wafers by infrared lock-in thermography," in Proceedings of the 17th European Photovoltaic Solar Energy Conference, pp. 1597-1599, WIP, Munich, Germany, October 2001.

[11] P. Pohl, J. Schmidt, K. Bothe, and R. Brendel, "Mapping of trap densities and energy levels in semiconductors using a lockin infrared camera technique," Applied Physics Letters, vol. 87, no. 14, Article ID 142104, 3 pages, 2005.

[12] W. Shockley and W. T. Read Jr., "Statistics of the recombinations of holes and electrons," Physical Review, vol. 87, no. 5, pp. 835-842, 1952.

[13] R. N. Hall, "Electron-hole recombination in germanium," Physical Review, vol. 87, no. 2, p. 387, 1952.

[14] J. Schmidt and R. A. Sinton, "Defect characterization by temperature and injection-dependent lifetime spectroscopy," in Proceddings of the 3rd World Conference on Photovoltaic Energy Conversion (WCPEC'03), pp. 947-950, Osaka, Japan, May 2003.

[15] J. Schmidt, P. Pohl, K. Bothe, C. Schmiga, R. Krain, and R. Brendel, "Advanced defect characterisation techniques in crystalline silicon-based photovoltaics," in Proceedings of the 21st European Photovoltaic Solar Energy Conference, pp. 524-529, WIP, Dresden, Germany, September 2006.

[16] T. Lauinger, J. Schmidt, A. G. Aberle, and R. Hezel, "Record low surface recombination velocities on $1 \Omega \mathrm{cm} \rho$-silicon using remote plasma silicon nitride passivation," Applied Physics Letters, vol. 68, no. 9, pp. 1232-1234, 1996.
[17] K. Graff, Metal Impurities in Silicon-Device Fabrication, Springer, Berlin, Germany, 2nd edition, 2000.

[18] S. Diez, S. Rein, and S. W. Glunz, "Analyzing defects in silicon by temperature- and injection-dependent lifetime spectroscopy (T-IDLS)," in Proceedings of the 20th European Photovoltaic Solar Energy Conference, pp. 1216-1219, WIP, Barcelona, Spain, June 2005.

[19] S. W. Glunz and W. Warta, "High-resolution lifetime mapping using modulated free-carrier absorption," Journal of Applied Physics, vol. 77, no. 7, pp. 3243-3247, 1995.

[20] O. Breitenstein and M. Langenkamp, Lock-in Thermography, Springer, Berlin, Germany, 2003.

[21] J. Isenberg and W. Warta, "Spatially resolved evaluation of power losses in industrial solar cells by illuminated lock-in thermography," Progress in Photovoltaics: Research and Applications, vol. 12, no. 5, pp. 339-353, 2004.

[22] T. Trupke, R. A. Bardos, M. C. Schubert, and W. Warta, "Photoluminescence imaging of silicon wafers," Applied Physics Letters, vol. 89, no. 4, Article ID 044107, 3 pages, 2006.

[23] P. Pohl and R. Brendel, "Temperature dependent infrared camera lifetime mapping (ILM)," in Proceedings of the 19th European Photovoltaic Solar Energy Conference, pp. 46-49, WIP, Paris, France, June 2004.

[24] P. Pohl, J. Schmidt, C. Schmiga, and R. Brendel, "Defect imaging in multicrystalline silicon using a lock-in infrared camera technique," Journal of Applied Physics, vol. 101, no. 7, Article ID 073701, 11 pages, 2007.

[25] D. K. Schroder, R. N. Thomas, and J. C. Swartz, "Free carrier absorption in silicon," IEEE Transactions on Electron Devices, vol. 25, no. 2, pp. 254-261, 1978.

[26] R. Bube, Photoelectronic Properties of Semiconductors, Cambridge University Press, Cambridge, UK, 1992.

[27] D. Macdonald and A. Cuevas, "Trapping of minority carriers in multicrystalline silicon," Applied Physics Letters, vol. 74, no. 12, pp. 1710-1712, 1999.

[28] J. Schmidt, K. Bothe, and R. Hezel, "Oxygen-related minoritycarrier trapping centers in $\rho$-type Czochralski silicon," Applied Physics Letters, vol. 80, no. 23, pp. 4395-4397, 2002.

[29] J. A. Hornbeck and J. R. Haynes, "Trapping of minority carriers in silicon-I. $\rho$-type silicon," Physical Review, vol. 97, no. 2, pp. 311-321, 1955.

[30] W. Warta, "Advanced defect and impurity diagnostics in silicon based on carrier lifetime measurements," Physica Status Solidi (A), vol. 203, no. 4, pp. 732-746, 2006.

[31] P. Pohl, J. Schmidt, K. Bothe, and R. Brendel, “Trap density imaging of silicon wafers using a lock-in infrared camera technique," in Proceedings of the 4th IEEE World Conference on Photovoltaic Energy Conversion, vol. 1, pp. 932-935, Waikoloa, Hawaii, USA, May 2006.

[32] W. C. Dash, "Copper precipitation on dislocations in silicon," Journal of Applied Physics, vol. 27, no. 10, pp. 1193-1195, 1956.

[33] M. Schubert, S. Riepe, and W. Warta, "Spatially resolved trapping detection and correlation with material quality in multicrystalline silicon," in Proceedings of the 21st European Photovoltaic Solar Energy Conference, pp. 629-633, WIP, Dresden, Germany, September 2006. 

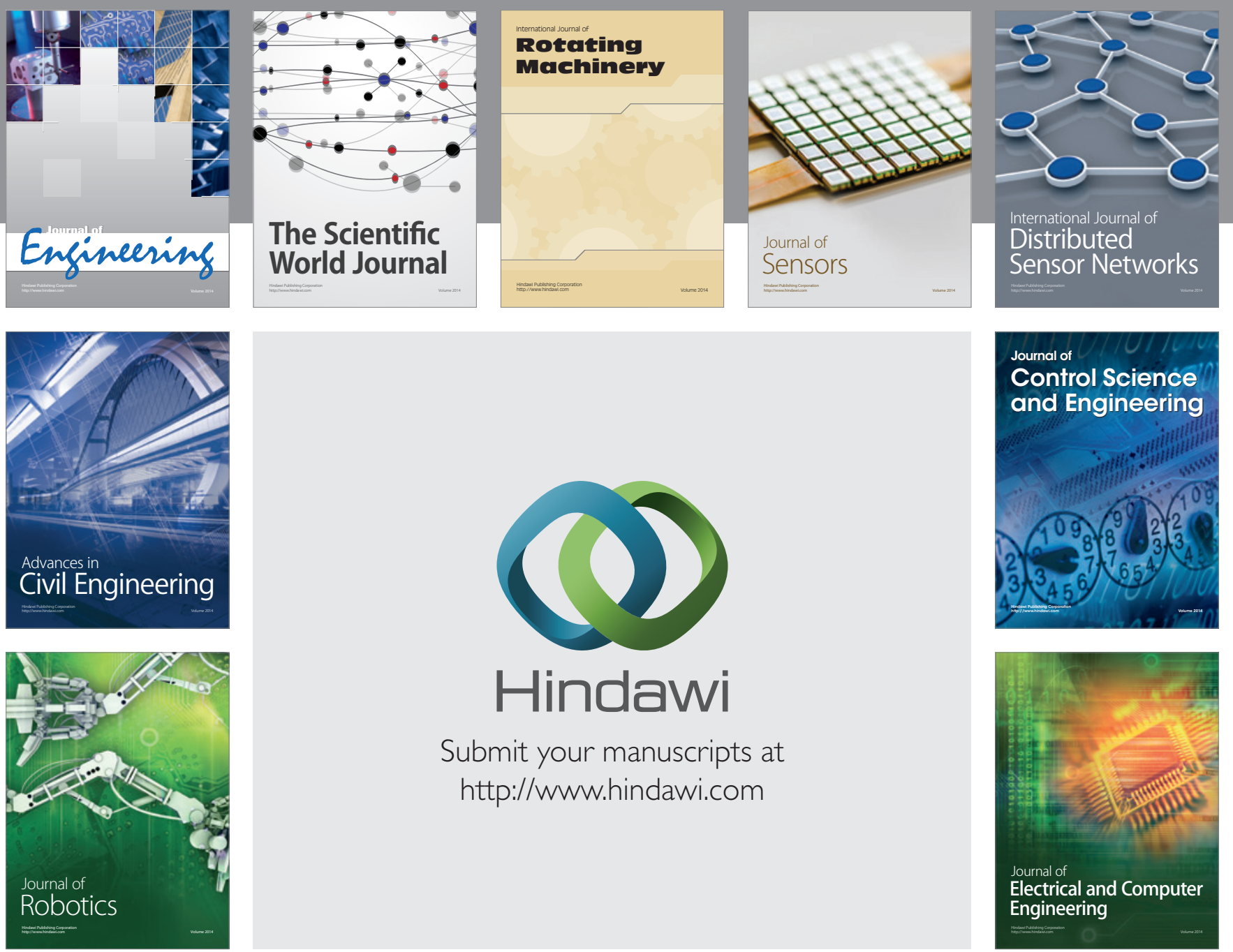

Submit your manuscripts at

http://www.hindawi.com
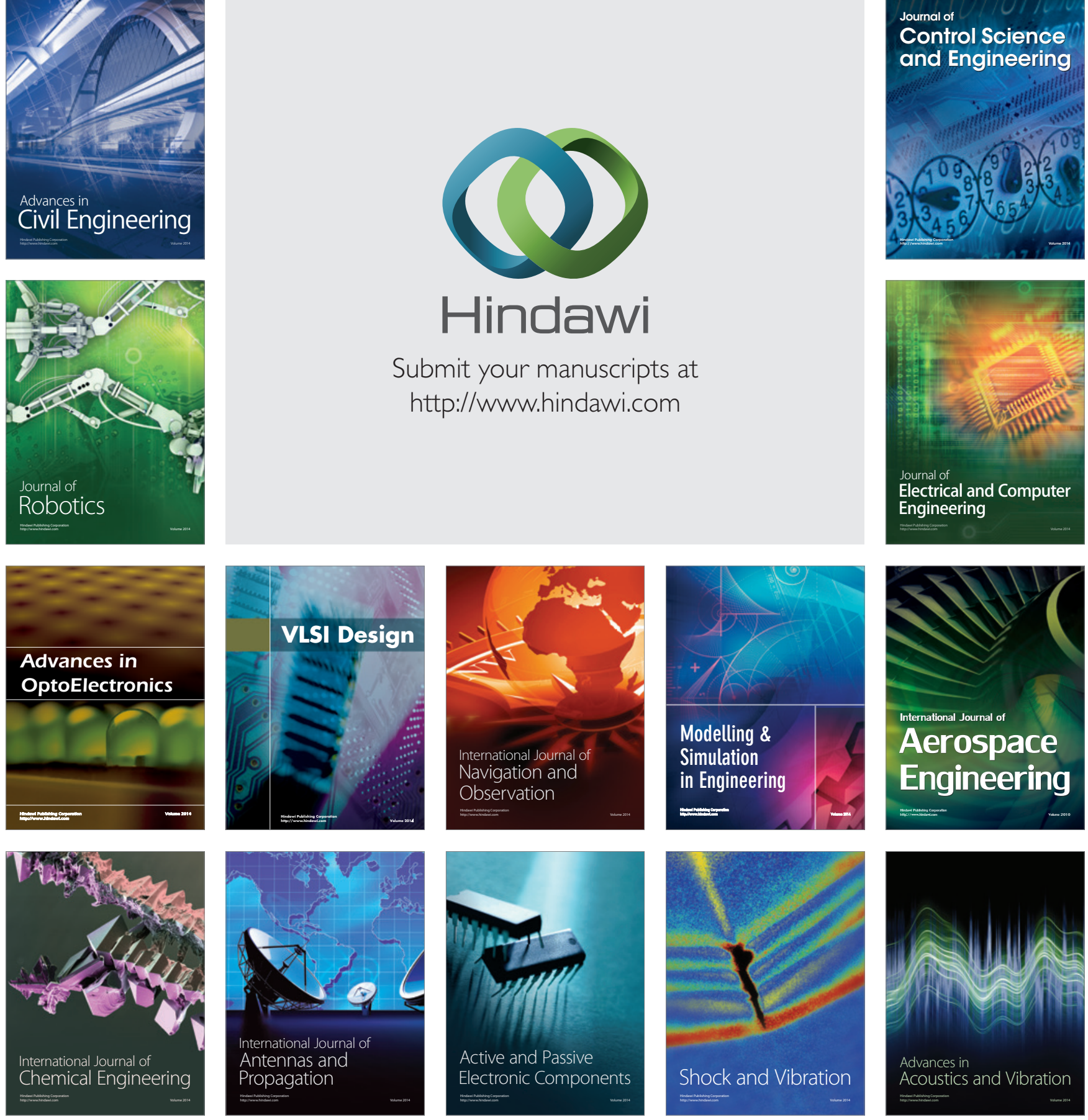